\title{
Efficacy of pelvic floor magnetic stimulation combined with electrical stimulation on postpartum pelvic organ prolapse: a retrospective study
}

\author{
(1) LIN ZHANG, (1) XIAO JUAN LV, (1) JIASONG TANG \\ Second Treatment Area, Department of Gynecology, Changchun Obstetrics-Gynecology Hospital, Jilin, China
}

\begin{abstract}
Objective: To evaluate the effect of pelvic floor magnetic stimulation combined with electrical stimulation on postpartum pelvic organ prolapse.

Materials and Methods: A retrospective study was performed by reviewing the clinical records of postpartum patients with pelvic organ prolapse (POP). POP stage, pelvic floor muscle (PFM) strength, and intrapelvic surface electromyography were compared before and after 20 treatment sessions of combined pelvic floor magnetic and electrical stimulation.

Results: A total of 90 patients' data files were analyzed. One-hundred percent (90 of 90) of the patients improved by decreasing one stage of POP; 93.3\% (84 of 90) of patients improved their PFM; the amplitudes for phasic, tonic and endurance contraction were all significantly increased.
\end{abstract}

Conclusions: The cocktail scheme combining magnetic stimulation, electrical stimulation and biofeedback, not only increases the awareness of PFM, but also improves the PFM contraction and POP stage.

Keywords: Electrical stimulation; pelvic floor magnetic stimulation; pelvic organ prolapse; postpartum

\section{INTRODUCTION}

Pelvic organ prolapse (POP) is a condition in which organs fall down or slip out of place and present as a bulge or herniation of a pelvic organ. POP is classified as a cystocele, rectocele, uterine prolapse, enterocele or vault prolapse. The prevalence of POP, based on a clinical evaluation, in general population of females was considered to be more than $30 \%$, while prevalence based on vaginal bulge symptoms ranged between 5 and $10 \%{ }^{1}$ Symptomatic POP may cause substantial discomfort, lower quality of life and restrict daily activities. An estimated lifetime cumulative risk of $7-11 \%$ POP surgery has been reported and reoperation is common. ${ }^{1}$ Prevention and diagnosis of early symptoms is important. The incidence rate of POP stage $\geq 11$ is estimated to be between 18-56\% 3 to 6 months' postpartum., ${ }^{2,3}$ Pregnancy, childbirth, and heavy lifting can weaken the structure of the vagina and cause POP. ${ }^{4}$

Treatment options vary depending on the severity of symptoms and the degree of prolapse. Conservative management includes lifestyle advice, pessary, pelvic floor muscle (PFM) training (PFMT), biofeedback, electrical stimulation, to mention some of the common interventions, and these are usually adopted for mild to moderate degrees of POP. Evidence suggests pessary and PFMT are also effective first-line treatments for women

Address for Correspondence: Jiasong Tang, Second Treatment Area, Department of Gynecology, Changchun Obstetrics-Gynecology Hospital, Jilin, China E-mail: 14883889@qq.com ORCID ID: orcid.org/0000-0002-7156-3914

Received: 11 February 2020 Accepted: 21 February 2020

(C) Copyright 2020 by the International Society for Pelviperineology / Pelviperineology published by Galenos Publishing House. 
with POP in stages I, II and asymptomatic stage III. Activity of the PFM plays a critical role in providing structural support to the pelvic organs. ${ }^{5}$ Several randomized controlled trials (RCTs) have shown that PFMT is effective in reducing symptoms and reversing or preventing further development of POP., 6 , PFMT can improve the performance, extension, and elasticity of the levator ani and perineal muscles. Furthermore, a blind RCT found that PFMT significantly improved PFM strength and thickness, elevated the bladder neck, narrowed the area of levator hiatus, and reduced the length of muscle in females with POP. These morphologic changes after PFMT reveal that strength training of PFM may potentially prevent or reverse postpartum POP.

The ability to perform a proper contraction of pelvic muscles is essential for PFMT to be effective. Most women don't know their pelvic floor, and it is estimated around $30-50 \%$ of women do not know how to contract their PFM correctly. ${ }^{8}$ Alternatively, most women activate and co-contract other muscles including the glutei, adductors, and abdomen, exhibit apnea, inhale too deeply, or trigger PFM to move downward (opposite to the desired action). Sometimes, it is enough for the therapist to provide verbal instruction on how to contract the muscles correctly, at other times, biofeedback or electrical/magnetic stimulation is necessary. Intravaginal electrical stimulation or magnetic stimulation can help women recognize and strengthen their PFM.

Electromyography (EMG) biofeedback provides instant, performance-dependent, visual and/or auditory feedback that measures the function of muscles and thus helps to increase self-consciousness and teach correct contraction of the muscle. The visual feedback is beneficial to the patient to learn how to upregulate underactive muscle or downregulate overactive muscle. ${ }^{9}$

In the pelvic floor area, electrical stimulation induces skeletal muscle training, remodels smooth muscle and/or connective tissues to enhance the pelvic floor support function. It helps to develop PFM awareness and is therapeutic for patients who present with PFM weakness. ${ }^{10}$

Magnetic stimulation relies on the use of electric current caused by a time-varying magnetic field. Depolarization of neural tissue tends to be distinct after either electrical or magnetic stimulation. Therefore, presumably, they affect the musculature of pelvic floor in a similar manner. Magnetic stimulation can result in hypertrophy of the PFM, change the proportions of type I and II fibers, and enable greater recruitment. In women with stress urinary incontinence (SUI) who can't isolate or contract their PFM sufficiently, the superiority of magnetic stimulation over sham therapy has been confirmed. ${ }^{11}$ Currently, despite the widespread use of magnetic stimulation in urinary incontinence, overactive bladder, constipation, there is no research on the effect of magnetic stimulation on postpartum POP.

A question that remains unanswered is whether patients can benefit from a combined therapy involving magnetic stimulation, biofeedback and electrical stimulation, rather than any one of these methods on its own? Although there is clear evidence that each of the strategies taken individually improves muscle strength, there is no research to test if the combination of these techniques used alternately produces better results than when carried out individually. The aim of this study was to evaluate the efficacy of pelvic floor magnetic stimulation combined with electrical stimulation and biofeedback on postpartum POP.

\section{MATERIALS AND METHODS}

This study is a retrospective study of patients from June 2018 to June 2019. Patients' clinical records were reviewed to gather information about each patient's presenting problem at their first postpartum checkup (from 42 days to 180 days after delivery), the treatment that was provided and the therapistassessed outcome at the final treatment session.

All patients who finished 20 sessions of treatment including combination of magnetic stimulation, electrical stimulation and biofeedback were enrolled in the group.

Inclusion criteria: age between 20-38 years; full-term pregnancy; single birth; POP-Q stage I or II.

Exclusion criteria: stages III or IV prolapse; with SUI or POP prior to pregnancy; third or fourth degree perineal tears; cancer in pelvic region; neurologic disorders; receiving other treatments for prolapse; serious illness to mother or child; any interruption for more than 2 weeks during treatment period; anyone with either a cardiac pacemaker or metallic hip implant.

\section{Intervention}

At the first postpartum check-up appointment (at day 42 or later), a standardized history was recorded and all women were educated in verbal and written form to perform PFMT. After they were taught how to contract their PFM correctly, all women underwent an assessment of PFM strength using a Modified Oxford scale. All participants undertook to do three sets of PFMT exercises daily for 8 weeks and were supervised during the 8 weeks' treatment.

\section{Treatment regimen}

The total treatment regimen included 20 sessions. The first five sessions were pelvic floor magnetic stimulation, three 
times per week. Then the patients practiced PFMT with the assistance of biofeedback from the sixth to tenth session, followed by 15 minutes electrical stimulation, three times per week. Then the eleventh, thirteenth, fifteenth, seventeenth and nineteenth session was magnetic stimulation and the twelfth, fourteenth, sixteenth, eighteenth and twentieth session was electrical stimulation combined with biofeed backassisted PFMT.

\section{Biofeedback-assisted PFMT}

The surface EMG (SEMG) biofeedback exercises were adopted to assist with PFMT. To record the PFM, a single channel SEMG vaginal probe (two longitudinal stainless electrodes on both sides) was used in connection with a Myotrac Infiniti (Thought Technology Ltd., Montreal, PQ, Canada). Two abdominal patch electrodes were attached to monitor any abdominal muscle co-contraction during PFM contraction. The rectified mean square sEMG signal from the intravaginal probe, monitoring pubococcygeus muscle, was displayed together with the abdominal tracing.

The double channel system is necessary to teach patients how to contract the PFM muscles without co-contracting abdominal muscles. The feedback screen was always set within the range of the individuals flick and tonic contraction, to train them to improve their ability of the coordination, strength and endurance of PFM. The biofeedback-assisted PFMT lasted 15 minutes per session.

\section{Electrical stimulation}

Electrical stimulation was delivered using the same instrument used for biofeedback-assisted PFMT. The placement of the vaginal probe was the same as previous description. The stimulus consisted of a fixed parameter asymmetric biphasic current: frequency $30 \mathrm{~Hz}$, pulse width $300 \mu \mathrm{s}$, work time/rest time $8 \mathrm{~s} / 10$ $\mathrm{s}$, ramp up $1 \mathrm{~s}$, ramp down $1 \mathrm{~s}$. Pulse intensity was regulated to the most tolerable intensity. Electrical stimulation session lasted 15 minutes.

\section{Pelvic floor magnetic stimulation}

During treatment, patients were seated fully clothed in an armchair of Magneuro60F(Nanjing Vishee Medical Ltd., Nanjing, jiangsu, China). Within the armchair's seat was a magnetic field generator (stimulation coil) which was connected to and controlled by the main engine. The treatment frequency of the pulsed magnetic field was $30 \mathrm{~Hz}$ and the treatment intervals were intermittent ( $5 \mathrm{~s}$ on and $5 \mathrm{~s}$ off). The amplitude (0-100\%) was adjusted to the most tolerable intensity. Treatment session lasted 20 minutes.

\section{Outcome measures}

The primary outcomes were measured as stage of POP. Secondary outcomes were PFM strength and intrapelvic sEMG readings.

\section{POP stage}

Stage of POP was diagnosed using the reliable POPQuantification examination (POP-Q) in which the defined maximal point of vaginal descent is measured relative to the hymen during strain with the woman in a supine lithotomy position. The POP-Q examination was conducted by a fixed gynecologist who followed a rigorous protocol and a standardized procedure.

\section{PFM strength}

PFM strength was measured using Modified Oxford Scale, performed using digital assessment. During digital examination, the examiner asked the woman to contract her PFM without any assistance from abdominal, hip and leg muscles, and then assigned a score out of 0-5 (0-no contraction, 1-flicker, 3-weak, 4-moderate, 5-strong).

\section{Intrapelvic sEMG assessment}

The Glazer Protocol was used for assessment of PFM. The protocol was developed by Glazer and Hacad ${ }^{12}$, and records and analyses the SEMG signal associated with the neuromuscular activation of PFM. The Glazer Protocol is composed of a fixed series of contraction and relaxation, directed via on-screen sEMG tracing templates and voice commands. The fixed sequence of muscular activity includes: $60 \mathrm{~s}$ pre-baseline rest, five phasic (quick flick) contractions with $10 \mathrm{~s}$ interval between each contraction (phasic contraction), five 10 sustained contractions with $10 \mathrm{~s}$ interval between each contraction (tonic contraction), $60 \mathrm{~s}$ endurance contraction, $60 \mathrm{~s}$ post-baseline rest. The EMG signal and data analysis were conducted using the Myotrac Infiniti and Bioneuro Infiniti. The amplitudes for phasic, tonic and endurance contraction were analysed.

\section{Statistical Analysis}

Data was analysed using SPSS, version 19. All data were presented as mean \pm standard deviation (SD) of the mean. Paired t-tests were conducted to compare the data of pre-treatment and posttreatment. P value was set to $\leq 0.05$.

\section{RESULTS}

A total of 90 patients met inclusion for this study. Of these women, $26.7 \%$ had stage I and $73.3 \%$ had stage II POP; $12.2 \%, 25.6 \%$, 
55.6\%, 6.6\% had degree 0, 1, 2 and 3 PFM muscle strength (Table 1). After 20 times of treatment, $100 \%$ of the patients improved by decreasing one stage of POP (Table 2). For improvement of PFM strength, only $6.7 \%$ patients' PFM strength didn't change. 93.3\% of patients improved their PFM. 10\% patients increase two degrees (Table 3). According to EMG values, after 20 treatment sessions, the amplitudes for phasic, tonic and endurance contraction were all significantly increased $(p<0.01)$ (Table 4$)$.

\section{DISCUSSION}

This study shows that a "cocktail" scheme (magnetic stimulation, electrical stimulation and biofeedback) is associated with significant improvement in the stage of postpartum POP, and PFM strength for postpartum women. After receiving two months of "cocktail" treatment, the patients with stage II POP recovered to normal or stage I POP, and enhanced PFM muscle strength. The EMG assessment showed a very significant change. Other researchers also reported significant improvement following 16-week of PFMT, plus lifestyle advice intervention, showing significant improvement in POP symptoms and POP-Q measurements, and $45 \%$ change in POP stage. ${ }^{13}$ PFMT appears to be crucial to the conservative management of POP, but an insufficient treatment dose or low adherence

\section{Table 1. The demographic characteristics of the participants}

\begin{tabular}{|c|c|c|}
\hline & & Value \\
\hline \multirow[t]{2}{*}{ Stage of POP } & Stage I & 24 \\
\hline & Stage II & 66 \\
\hline \multirow{4}{*}{ PFM strength } & 0 & 11 \\
\hline & 1 & 23 \\
\hline & 2 & 50 \\
\hline & 3 & 6 \\
\hline Age (mean \pm SD) & - & $29.50 \pm 3.58$ \\
\hline Vaginal delivery number & - & 80 \\
\hline Cesarean delivery & - & 10 \\
\hline BMI (mean \pm SD) & - & $23.18 \pm 3.12$ \\
\hline
\end{tabular}

POP: pelvic organ prolapse, PFM: Pelvic floor muscle, SD: Standard deviation, BMI: Body mass index

Table 2. Change in severity stage from pre-treatment to post-treatment

\begin{tabular}{|l|l|}
\hline Change in stage & People $(\mathbf{n}=\mathbf{9 0})$ \\
\hline From stage I to 0 & 24 \\
\hline From stage II to I & 66 \\
\hline -1 stage & 90 \\
\hline n: Number & \\
\hline
\end{tabular}

$\begin{aligned} & \text { Table 3. Change in PFM } \\
& \text { post-treatment }\end{aligned}$
\begin{tabular}{|l|l|} 
Change in PFM strength & People $(\mathbf{n}=\mathbf{9 0})$ \\
\hline+2 degrees & 9 \\
\hline+1 degree & 77 \\
\hline No change in degree & 6 \\
\hline PFM: Pelvic floor muscle, n: Number
\end{tabular}

Table 4. Change in intrapelvic sEMG value between baseline, 6 weeks' postpartum and post-treatment $(n=90)$

\begin{tabular}{|l|l|l|l|}
\hline & $\begin{array}{l}\text { Pre- } \\
\text { treatment } \\
(\boldsymbol{\mu V})\end{array}$ & $\begin{array}{l}\text { Post- } \\
\text { treatment } \\
(\boldsymbol{\mu V})\end{array}$ & $\mathbf{p}$ \\
\hline Phasic contractions & $18.87 \pm 8.65$ & $31.48 \pm 6.66$ & $1.30 \mathrm{E}-21$ \\
\hline Tonic contractions & $13.17 \pm 6.17$ & $29.53 \pm 4.40$ & $1.32 \mathrm{E}-45$ \\
\hline Endurance contractions & $12.84 \pm 6.15$ & $27.55 \pm 3.76$ & $1.22 \mathrm{E}-45$ \\
\hline \multicolumn{3}{|l}{ sEMG: Surface electromyography } \\
\hline
\end{tabular}

to training will significantly decrease the improvement rate. Biofeedback was originally introduced to treat urinary incontinence, constipation, overactive bladder and chronic pelvic pain. The basic goal is to make patients aware of their muscle function and enhance the quality of PFMT. However, it has been shown to be an effective approach to increase the PFM strength and alleviate the symptoms of POP. ${ }^{14}$ Electrical stimulation is commonly used when rehabilitating muscles because it activates nerve fibers. Pelvic floor electrical stimulation has been used to stimulate the pudendal nerve for PFM activation. Intravaginal electrical stimulation may help patients identify and contract their PFM, strengthening these muscles and substantially assisting with pelvic floor dysfunction. ${ }^{15}$ Electrical stimulation is used separately or together with PFMT to alleviate urinary incontinence (UI) symptoms, but it is seldom put to use in POP treatment, yet its impact has been confirmed in PFM strength improvement. Magnetic stimulation therapy is a new way of providing noninvasive, passive stimulation to the pelvic floor. In 1998, the United States Food and Drug Administration approved this new form of conservative therapy for UI. An electrical coil generates pulsed magnetic fields which generate an induced ion surge, at the tissue level. The electrical eddy currents lead to depolarization of the membrane. It allows PFM to contract and in time the training leads to a reduction of UI symptoms. Magnetic stimulation alleviated UI frequency, improved micturition and quality of life of UI patients. In particular, patients who may not have the ability to do normal PFMT can be treated using this method.

Culligan's team performed a randomized, double-blinded, 
sham-controlled study of postpartum extracorporeal magnetic innervations to restore pelvic muscle strength at six weeks' postpartum. They found that using eight weeks of treatment (twice weekly) with $50 \mathrm{~Hz}$ magnetic stimulation for $20 \mathrm{~min} /$ session to regain PFM strength after childbirth as ineffective. ${ }^{16}$ However, in present research the combination of magnetic and electrical stimulation resulted in a significant increase in PFM strength. The different results may be due to: (1) treatment protocol; in Culligan's trial, all patients only received magnetic stimulation, whereas in the present study, we combined magnetic stimulation, electric stimulation and biofeedback together. Biofeedback is useful to help patients master how to contract PFM correctly and efficiently, improving their score when practicing PFM strength assessment; (2) the frequency of $50 \mathrm{~Hz}$ chosen in Culligan's trial is not optimal for strengthening PFM. In the present study, $30 \mathrm{~Hz}$ is used for both electrical and magnetic stimulation.

\section{Study Limitations}

The limitation of this study is that there was no control group. RCTs are needed before drawing any definitive conclusions on the effect of pelvic floor magnetic stimulation combined with electrical stimulation in prevention and treatment on postpartum POP.

\section{CONCLUSIONS}

This is the first study to report a cocktail scheme for postpartum POP management. For young women who suffer POP after vaginal or cesarean section delivery, rehabilitation of the PFM is important in the prevention and treatment of POP according to Norton's dry boat theory. But for some reasons, the homebased PFMT is not a good recommendation for postpartum women in China due to the special postpartum care culture. Chinese postpartum women are always concerned about the new babies so that it's common for them to forget to practice PFMT. Forgetting to do PFMT is the main cause of low adherence. Therefore, development of a high adherence treatment scheme is important for them. The cocktail scheme combining magnetic stimulation, electrical stimulation and biofeedback, not only increases the awareness of PFM, but also improves the PFM contraction, finally improves the POP stage.

\section{DISCLOSURES}

The author declares no conflict of interest, no financial support by any grant or research sponsor, and no competing financial interest.

\section{Authorship Contributions}

Medical Practices: Lin Zhang, Xiaojuan Lv, Concept: Jiasong Tang, Design: Jiasong Tang, Data Collection or Processing: Lin Zhang, Xiaojuan Lv, Analysis or Interpretation: Lin Zhang, Literature Search: Lin Zhang, Writing: Jiasong Tang.

Peer-review: Externally peer-reviewed.

\section{REFERENCES}

1. Milsom I, Altman D, Cartwright R, et al. Epidemiology of urinary incontinence (UI) and other lower urinary tract symptoms (LUTS), pelvic organ prolapse (POP) and anal incontinence (AI). In: Incontinence: 5th International Consultation on Incontinence, Paris: 2012 ICUD-EAU, 2013. p. 15-107.

2. Diez-Itza I, Arrue M, Ibanez L, Paredes J, Murgiondo A, Sarasqueta C. Influence of mode of delivery on pelvic organ support 6 months postpartum. Gynecol Obstet Invest 2011; 72: 123-9.

3. Diez-Itza I, Arrue M, Ibanez L, Paredes J, Murgiondo A, Sarasqueta C. Postpartum impairment of pelvic floor muscle function: factors involved and association with prolapse. Int Urogynecol J 2011; 22: 1505-11.

4. Gyhagen M, Bullarbo M, Nielsen TF, Milsom I. Prevalence and risk factors for pelvic organ prolapse 20 years after childbirth: a national cohort study in singleton primiparae after vaginal or caesarean delivery. BJOG 2013; 120: 152-60.

5. Braekken IH, Majida M, Ellström Engh M, Holme IM, Bø K. Pelvic floor function is independently associated with pelvic organ prolapse. BJOG 2009; 116: 1706-14.

6. Stüpp L, Resende AP, Oliveira E, et al. Pelvic floor muscle training for treatment of pelvic organ prolapse: an assessor-blinded randomized controlled trial. Int Urogynecol J 2011; 22: 1233-9.

7. Bø K, Hilde G, Stær-Jensen J, Siafarikas F, Tennfjord MK, Engh ME. Postpartum pelvic floor muscle training and pelvic organ prolapse-a randomized trial of primiparous women. Am J Obstet Gynecol 2015; 212: 38.

8. Rodrigues MP, Barbosa LJF, Paiva LL, et al. Effect of intravaginal vibratory versus electric stimulation on the pelvic floor muscles: A randomized clinical trial. Eur J Obstet Gynecol Reprod Biol X 2019; 3: 100022.

9. Fitz FF, Resende AP, Stüpp L, Sartori MG, Girão MJ, Castro RA. Biofeedback for the treatment of female pelvic floor muscle dysfunction: a systematic review and meta-analysis. Int Urogynecol J 2012; 23: 1495-516.

10. Kannan P, Winser SJ, Fung B, Chening G. Effectiveness of Pelvic Floor Muscle Training Alone and in Combination With Biofeedback, Electrical Stimulation, or Both Compared to Control for Urinary Incontinence in Men Following Prostatectomy: Systematic Review and Meta-Analysis. Phys Ther 2018; 98: 932-45.

11. Peng L, Zeng X, Shen H, Luo DY. Magnetic stimulation for female patients with stress urinary incontinence, a meta-analysis of studies with short-term follow-up. Medicine (Baltimore) 2019; 98: 15572.

12. Glazer HI, Hacad CR. The Glazer Protocol: evidence-based medicine pelvic floor muscle (PFM) surface electromyography (SEMG). Biofeedback 2012; 40: 75-9. 
13. Hagen S, Stark D, Glazener C, Sinclair L, Ramsay I. A randomized controlled trial of pelvic floor muscle training for stages I and II pelvic organ prolapse. Int Urogynecol J Pelvic Floor Dysfunct 2009; 20: 45-51.

14. Geris MGS, Elkosery SM, Azzam HAM. Effect of biofeedback and pelvic floor muscles training on genital prolapse. Drug Invention Today 2019; 12: 2950-9.

15. Abdelbary AM, El-Dessoukey AA, Massoud AM, et al. Combined Vaginal Pelvic Floor Electrical Stimulation (PFS) and Local
Vaginal Estrogen for Treatment of Overactive Bladder (OAB) in Perimenopausal Females. Randomized Controlled Trial (RCT). Urology 2015; 86: 482-6.

16. Culligan PJ, Blackwell L, Murphy M, Ziegler C, Heit MH. A randomized, double-blinded, sham-controlled trial of postpartum extracorporeal magnetic innervation to restore pelvic muscle strength in primiparous patients. Am J Obstet Gynecol 2005; 192: 1578-82. 